\title{
Born during Maternal Ketoacidosis; Recognition of Neonatal Consequences
}

\section{Lauren C Weeke* and Van Straaten HLM}

Department of Neonatology, Isala Clinics, Zwolle, The Netherlands

\begin{abstract}
After peripartum asphyxia, metabolic acidosis and neurological derangement are clinical findings. In severe cases, treatment with hypothermia should be started within six hours after birth. A rare cause of neonatal metabolic acidosis is maternal metabolic derangement due to Diabetic Keto Acidosis (DKA).

We report characteristics of a boy born at $336 / 7$ weeks gestation to a mother with unrecognized DKA in gestational diabetes mellitus. Persistent metabolic acidosis and severe neurological derangement resulted. Diagnosed as severe peripartum asphyxia, hypothermia was started. Hours after delivery, the mother was diagnosed with DKA. Although the child's diagnosis of peripartum asphyxia was revised, hypothermia was continued.

In newborns with severe metabolic acidosis and normal serum lactate, maternal metabolic derangement due to DKA should be considered. In those cases hypothermia is not proven effective. Early recognition and treatment of maternal DKA potentially prevents fetal death, neonatal sequelae and in less severe cases, unnecessary invasive hypothermic therapy.
\end{abstract}

Keywords: Diabetes mellitus; Diabetic ketoacidosis; Pregnancy; Neonatal; Asphyxia

Abbreviations: DKA -Diabetic Ketoacidosis; GDM - Gestational Diabetes Mellitus

\section{Introduction}

Metabolic acidosis directly postpartum is a common feature in the (pre-) term neonate. The number one cause of metabolic acidosis shortly after delivery is peripartum asphyxia. In severe cases, hypothermia is the method of treatment to be started within six hours after birth. Other, less common, causes include: shock, hypothermia, diabetes mellitus, renal insufficiency and inborn errors of metabolism.

A rare cause of neonatal metabolic acidosis is maternal metabolic derangement due to Diabetic Keto Acidosis (DKA). DKA occurs in $1-10 \%$ of pregnancies complicated by pregestational diabetes mellitus, but it is rarely seen in Gestational Diabetes Mellitus (GDM) (incidence $0.45 \%$ ) [1-3]. In pregnancy, DKA tends to occur at lower glucose levels and a small percentage (0.8-1.1\%) occurs at normal glucose levels $(\leq 10$ $\mathrm{mmol} / \mathrm{L})[1,4]$. Other symptoms of DKA are nausea, vomiting, dysand tachypnoea. Therefore, DKA is difficult to recognize in pregnant women and often diagnosed at a late stage. Little is known about neonatal sequelae of maternal DKA. In the newborn, clinical signs of DKA mimic those of asphyxia and (septic) shock, making it difficult to distinguish between the different diagnoses [5]. However, all require a different therapeutic approach.

In this case report we report a preterm infant with metabolic acidosis as a result of unrecognized maternal DKA, treated invasively as peripartum asphyxia.

\section{Case}

A male infant was born at $33^{6} / 7$ weeks gestation to a mother with GDM. Maternal diabetes was managed with a diet. Glucose control was suboptimal as shown by an $\mathrm{HbAlc}$ level of $47 \mathrm{mmol} / \mathrm{mol}$ (normal in pregnancy: $\leq 44 \mathrm{mmol} / \mathrm{mol}$ ) four days prior to delivery. Approximately 24 hours before delivery, the mother presented to the referring hospital with a 6-hour history of nausea, vomiting and sub febrile temperature. Blood glucose levels were 5.6-7.8 mmol/L (normal: 4.0-7.8 mmol/L). Bacterial infection was suspected (marked leukocytosis and elevated
C-reactive protein) and the mother was started on IV antibiotics. Although blood pressure was normal, pre-eclampsia was suspected based on hyperreflexia and treatment with IV magnesium sulphate was initiated. Fetal heart rate monitoring was normal at admission but revealed recurrent decelerations the next morning and a caesarean section was performed.

A boy (3020 grams, + 1.5 SD) was born with Apgar-scores of 6 and 8 recorded at 1 and 5 minutes respectively. Shortly after birth the infant showed signs of severe circulatory and respiratory collaps, treated with cardiorespiratory support (i.e. mechanical ventilation and dopamine infusion). Hypoglycemia $(1.3 \mathrm{mmol} / \mathrm{L})$ was treated with IV glucose. In addition, the child had signs of significant neurological derangement resulting in a high Thompson score $>7$ (score of 9). Combined with an umbilical arterial $\mathrm{pH}$ of 6.85 and a base excess of $-22 \mathrm{mmol} / \mathrm{L}$ the diagnosis of peripartum asphyxia was made and the infant was transferred to our hospital's Neonatal Intensive Care Unit for hypothermic therapy. As bacterial infection could not be ruled out, IV antibiotics were administered.

After transfer to our hospital, laboratory testing revealed a metabolic acidosis ( $\mathrm{pH} 7.21$, bicarbonate $16.3 \mathrm{mmol} / \mathrm{L}$, base excess $-10.3 \mathrm{mmol} / \mathrm{L}$, serum lactate $3.0 \mathrm{mmol} / \mathrm{L}$ ) considered as a result of peripartum asphyxia. Hypothermia was initiated within six hours after birth.

After delivery, the maternal situation deteriorated with dys- and tachypnoea. Laboratory testing showed metabolic acidosis ( $\mathrm{pH} 7.23$, $\mathrm{pCO}_{2} 1.9 \mathrm{kPa}$, bicarbonate $6 \mathrm{mmol} / \mathrm{L}$, base excess $\left.-20.6 \mathrm{mmol} / \mathrm{L}\right)$ with

*Corresponding author: Lauren C Weeke MD, Isala Clinics, Neonatology, P.O Box 104008000, GK Zwolle, The Netherlands, Tel: +31-38-4245055; Fax: +31-384247667; E-mail: I.weeke@isala.nl

Received August 10, 2012; Accepted August 25, 2012; Published August 27 2012

Citation: Weeke LC, Van Straaten HLM (2012) Born during Maternal Ketoacidosis Recognition of Neonatal Consequences. J Clin Case Rep 2:191. doi:10.4172/21657920.1000191

Copyright: ( $) 2012$ Weeke LC, et al. This is an open-access article distributed under the terms of the Creative Commons Attribution License, which permits unrestricted use, distribution, and reproduction in any medium, provided the original author and source are credited. 
a high anion gap and ketosis $(4.0 \mathrm{mmol} / \mathrm{L})$ (normal: $<0.1 \mathrm{mmol} / \mathrm{L})$. Serum lactate was within the normal range $(1.3 \mathrm{mmol} / \mathrm{L})$ (normal: 0.5-1.7 mmol/L). The mother was diagnosed with DKA five hours after delivery. This information did not reach us timely.

Hypothermia was already initiated for several hours. Although ketoacidosis due to maternal metabolic derangement was now the most likely diagnosis, it was decided to continue hypothermia for 72 hours.

The child was kept on ventilatory support for six days, but with low peak inspiratory pressure. Dopamine infusion could be discontinued after one day. Antibiotics were continued for five days, but C-reactive protein never exceeded $4 \mathrm{mg} / \mathrm{L}$. During hospital stay no seizures were observed and EEG was normal without signs of epileptiform activity. MRI of the brain on day-of-life five demonstrated punctate hemorrhagic lesions not consistent with typical hypoxic-ischemic injury. Followup imaging at full term (38 weeks gestation corrected) revealed small bilateral lesions occipitally after previous hypoxic-ischemic injury. At six months follow-up the infant exhibited normal neurodevelopment.

\section{Discussion}

This case revealed that in newborns with severe postpartum metabolic acidosis but normal serum lactate, maternal metabolic derangement due to DKA should be considered. Especially, when there is a history of diabetes mellitus or GDM.

DKA is characterized by hyperglycemia, metabolic acidosis with a high anion gap and positive serum and urine ketones, as a result of an absolute or relative insulin deficiency $[1,6]$. In pregnancy, DKA can occur at lower glucose levels because of: physiologic hemodilution, increased glucose utilization by the fetus and placenta and reduced buffering capacity due to physiologic respiratory alkalosis lowering serum bicarbonate concentration $[4,7,8]$.

Little is known about neonatal sequelae of maternal DKA; fetal demise is high, although varied incidence numbers have been reported (9\% to $90 \%$ ), and, to our knowledge, only one report on neonatal complications, linking maternal ketoacidosis and subsequent neonatal brain injury, has been published [8,9]. Previous studies have shown that ketoacids cross the placenta and may be toxic to the fetus. Also, maternal acidosis leads to decreased uteroplacental blood flow and decreased amount of oxygen available to the fetus due to increased affinity of maternal hemoglobin for oxygen $[2,10]$, with fetal hypoxia as a result. Also, glucose uptake by the fetal brain is diminished with (maternal) acidosis, leading to brain injury [9].

Hypothermic therapy is not indicated in these cases but early diagnosis of maternal DKA is important. With timely and appropriate treatment, fetal death and preterm labor can be prevented $[1,3,4]$. Therefore, in pregnant women with a history of diabetes mellitus or GDM routinely blood gas analysis should be advised when presenting with symptoms suggestive of DKA, such as nausea, vomiting, dys- and tachypnoea, even when blood glucose levels are normal.

This case was unique because at initial assessment of the child, we were unaware of the maternal situation since the diagnosis of DKA in the mother was made several hours after delivery. The child's symptoms were consistent with peripartum asphyxia (fetal heart rate decelerations, umbilical $\mathrm{pH}<7.0$ and Thompson score $>7$ ) and the key issue for neonatal diagnosis was the information on the mother's medical situation. Retrospectively however, a few parameters were indicative of a diagnosis other than peripartum asphyxia. Firstly, the Apgar-score was relatively good and the child had not needed immediate resuscitation. Secondly, the child demonstrated persistent severe metabolic acidosis with a serum lactate not exceeding $3.0 \mathrm{mmol} / \mathrm{L}$, indicating that the acidosis was due to an acid other than lactate. Lastly, the Thompsonscore might have been influenced by hypoglycemia present at time of assessment, making it an unreliable measure of encephalopathy.

The presented case underlines that in newborns with metabolic acidosis but with normal serum lactate, maternal metabolic derangement due to DKA should be considered. In those cases, hypothermia is not the preferred treatment. Early recognition of maternal DKA potentially prevents, not only fetal death, preterm labor and post-partum neonatal sequelae, but in less severe cases also unnecessary invasive hypothermic therapy.

\section{References}

1. Oliver R, Jagadeesan P, Howard RJ, Nikookam K (2007) Euglycemic diabetic ketoacidosis in pregnancy: An unusual presentation. J Obstet Gynaecol 27: 308

2. Parker JA, Conway DL (2007) Diabetic ketoacidosis in pregnancy. Obste Gynecol Clin North Am 34: 533-543.

3. Franke B, Carr D, Hatem MH (2001) A case of euglycemic diabetic ketoacidosis in pregnancy. Diabet Med 18: 858-859.

4. Guo RX, Yang LZ, Li LX, Zhao XP (2008) Diabetic Ketoacidosis in pregnancy tends to occur at lower blood glucose levels: case-control study and a case report of euglycemic ketoacidosis in pregnancy. J Obstet Gynecol Res 34: 324 330.

5. Abacı A, Razi CH, Ozdemir O, Hızlı S, Kıslal F, et al. (2010) Neonatal diabetes mellitus accompanied by diabetic ketoacidosis and mimicking neonatal sepsis a case report. J Clin Res Pediatr Endocrinol 2: 131-133.

6. Tarif N, Al Badr W (2007) Euglycemic diabetic ketoacidosis in pregnancy Saudi J Kidney Dis Transpl 18: 590-593.

7. Clark JD, McConnell A, Hartog M (1991) Normoglycemic ketoacidosis in woman with gestational diabetes. Diabet Med 8: 388-389.

8. Chico M, Levine SN, Lewis DF (2008) Normoglycemic diabetic ketoacidosis in pregnancy. J Perinat 28: 310-312.

9. Stenerson MB, Collura CA, Rose CH, Lteif AN, Carey WA (2011) Bilateral basa ganglia infarctions in a neonate born during maternal ketoacidosis. Pediatrics 128: e707-e710.

10. Chauhan SP, Perry KG Jr, McLaughlin BN, Roberts WE, Sullivan CA, et al. (1996) Diabetic ketoacidosis complicating pregnancy. J Perinat 16: 173-175. 